\title{
Using a Variation of Empirical Mode Decomposition To Remove Noise From Signals
}

\author{
M. F. Kaleem, A. Guergachi, S. Krishnan \\ Ryerson University, Toronto, Canada. \\ m2kaleem@ryerson.ca
}

\author{
A. E. Cetin \\ Bilkent University, Ankara, Turkey. \\ cetin@bilkent.edu.tr
}

\begin{abstract}
This paper will describe the application of $\tau$-based decomposition, which is a variation of the empirical mode decomposition method based on modified peak selection, to de-noising and de-trending of signals. The $\tau$-based decomposition method will be explained, and its application to synthetic and real-world signals in the context of de-noising and de-trending will be described. Comparison between the computational simplicity of the $\tau$-based decomposition method to denoising and de-trending of signals and approaches based on empirical mode decomposition will be highlighted.
\end{abstract}

Keywords: De-noising, de-trending, empirical mode decomposition.

\section{INTRODUCTION}

Empirical Mode Decomposition (EMD) is a technique for decomposing non-stationary and non-linear multi-component signals into amplitude and frequency modulated waveforms called intrinsic mode functions (IMFs), which are obtained by adaptively extracting all the oscillatory modes present in the signal. EMD is defined by an algorithm [1], and the extraction of IMFs is through a process called sifting. The sifting process involves approximating the local average of the signal to be decomposed. For this, upper and lower envelopes for the signal are formed using natural cubic splines through the maxima and minima (extrema), which are identified by locating the peaks (upper and lower) in the signal. The local average is then approximated as the mean of the envelopes.

The work presented in this paper is based on a variation of EMD, which we call $\tau$-based decomposition. In $\tau$-based decomposition, envelopes are formed using $\tau$-based peak selection, where $\tau$ represents a length of time from within which only the extrema with the maximum value is selected. The change in the choice of extrema limits the time scale over which the sifting process allows a frequency component in the signal to pass un-decomposed. Based on different values of $\tau$, different decompositions of a signal are possible. We have studied the performance of $\tau$-based decomposition, using different test signals, both synthetic and real-world. This allowed us to test separation of different frequency components in a signal into what we term as $\tau$-functions, based on different values of $\tau$. Importantly, we have empirically established a relation between the value of $\tau$ and the value of the frequency component that can be decomposed into a $\tau$-function using $\tau$-based decomposition.

In this paper, we will describe application of $\tau$-based decomposition to de-noising and de-trending of signals. By appropriate selection of the value of $\tau$, noise can be easily separated from the signal. Similarly, $\tau$-based decomposition provides a simple mechanism for de-trending of signals. Additionally, $\tau$-based decomposition allows separating the signals from noise in situations where the noise is in the form of transients. Such situations are categorized as intermittency in the signal, which occurs when components of particular frequencies either come into existence, or disappear from a signal, over short time durations. Intermittency negatively affects the EMD algorithm, giving rise to a well-known issue with EMD known as mode-mixing
[4]. Mode-mixing is said to occur when an IMF contains different frequency components which cannot be properly separated by the sifting process in EMD due to intermittency in the signal to be decomposed.

In this context, Section II will describe the $\tau$-based decomposition method. Section III will explain the limit that has been established for $\tau$. Application of $\tau$-based decomposition to de-noising and detrending of signals, both synthetic and real-world, will be described in Section IV. Finally, Section V will conclude the paper.

\section{II. $\tau$-BASED DECOMPOSITION}

$\tau$-based decomposition uses the sifting process to decompose a signal. However, instead of using a time-scale based on successive extrema, as is done in the case of EMD, a criterion for choosing the extrema based on a period $\tau$ is used. This criterion is explained next:

1) For a given signal $x(t)$, a time period denoted by $\tau$ is chosen, and for each interval $\tau$ over the whole signal, the highest from among the minima and maxima within $\tau$ are selected.

2) The upper envelope $E_{n(U)}$ is calculated by using a cubic spline to connect all the maxima identified (one maxima or peak per $\tau$ ). Similarly, the lower envelope $E_{n(L)}$ is calculated by connecting the identified minima.

3 ) The mean $E_{n(\text { mean) }}$ of the upper and lower envelopes is calculated, and $x(t)$ is updated by subtracting the mean from it $x(t) \leftarrow x(t)-E_{n(\text { mean })}$.

4) Since the previous 3 steps are based on the EMD algorithm, which has a stopping criteria built-in, these steps are continued till the algorithm stops and the signal $x(t)$ is decomposed into what we term as $\tau$-functions.

In the EMD-based sifting, step 3 is continued till $x(t)$ is reduced to an IMF, which then is subtracted from $x(t)$ to get the residue $r_{1}(t)$. The residue $r_{1}(t)$ is then taken as the starting point instead of $x(t)$, and previous steps of the algorithm are repeated to find all the IMFs in the signal. In contrast to this, $\tau$-based peak selection reduces the signal $x(t)$ to coarse-grained $\tau$-functions, which may contain different coexisting modes of oscillation, each superimposed on the other. This happens because the choice of extrema based on the period $\tau$ limits the scale over which the sifting process allows component(s) to pass. In this context, $\tau$ sets an upper limit on the periods of the oscillations that can be included in any given $\tau$-function obtained using $\tau$-based decomposition. In section III we will describe this limit.

Limiting the number of extrema over the length of the signal, however, as would happen with a large $\tau$, can negatively affect the formation of accurate envelopes using cubic splines. To overcome this, we use the sifting approach of Ensemble Empirical Mode Decomposition (EEMD) [3], which is a noise-assisted version of EMD. In EEMD, a white noise series is added to the signal to be decomposed. In general, the amplitude of the white noise, $\sigma$, is 
kept around 0.2 times the standard deviation of the original signal. Then, using the sifting algorithm, the signal with the added noise is decomposed into IMFs. The previous two steps are repeated $N$ times, with a different white noise series each time. The final set of IMFs is obtained by calculating the ensemble means of the IMFs $c_{j}(t)$ obtained by the decompositions. This can be seen as:

$$
c_{j}(t)=\lim _{N \rightarrow \infty} \frac{1}{N} \sum_{k=1}^{N} c_{j k}(t)
$$

As described in [3], the white noise series added to the signal during the decomposition process cancel each other out when the ensemble mean of the IMFs is calculated according to Eq. 1. For the $\tau$-based decomposition method, using the EEMD approach proves useful with the formation of accurate envelopes using cubic splines, specially for the cases where only a few peaks are selected given a large $\tau$. This happens because the addition of a noise series provides peaks throughout the length of the signal, whatever the $\tau$ used, and hence accurate envelope formation is possible, leading to accurate $\tau$ functions. This is in contrast to EMD, where using a large $\tau$ would lead to few peaks being selected, and hence inaccurate envelope formation using cubic splines.

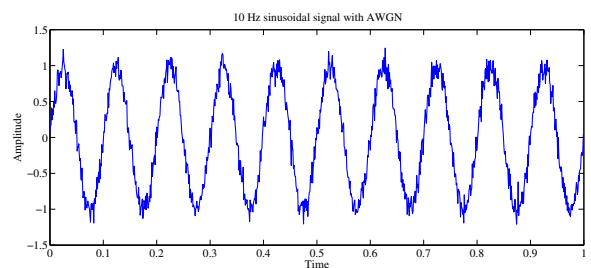

Fig. 1. Low frequency sinusoidal signal with additive white gaussian noise and $\mathrm{SNR}=20 \mathrm{~dB}$

\section{A LIMIT FOR $\tau$}

The value of $\tau$ limits the scale over which a component is allowed to pass. This scale is determined by $\frac{F_{s}}{\tau}$, where $F_{s}$ is the sampling frequency, e.g. a value of $\tau=40$ (in samples) corresponds to a frequency value $F=25 \mathrm{~Hz}$ for a $F_{s}=1000 \mathrm{~Hz}$. Using this $\tau$, only one peak in each 40 sample interval would be used in the envelope formation, and the sifting process should then decompose all $F \leq 25$ components, and let all components with $\mathrm{F}>25 \mathrm{~Hz}$ pass through un-decomposed in one $\tau$-function. This would suggest that in order to isolate a frequency component $F_{n}$, corresponding to a period $T_{n}$, a value of $\tau \leq x T_{n}$ is needed, where $0<x \leq 1$. Through experiments with different signals, and with different values of $\tau$, we have been able to establish a value of $\tau$ which can be written as

$$
0.5 T_{n}<\tau<0.63 T_{n}
$$

or equivalently

$$
1.6 F_{n}<F_{\tau}<2 F_{n}
$$

where $F_{\tau}$ corresponds to the frequency value represented by the value of $\tau$.

Equations 2 and 3 hold in general for a wide variety of signals. This means that for a signal of frequency $10 \mathrm{~Hz}$ mixed with additive noise, which has been sampled at 1000 samples per second, $\tau=50$ is a good value to separate the signal from the noise. According to Eq. 3, this value of $\tau$ corresponds to twice the value of the frequency $F_{n}$ of the component. Depending on the type of signals, at times it might be necessary to use a value of $\tau$ more than that established in Eqs. 2 or 3. This is necessitated due to the use of EEMD and is explained in Sec. IV-A.
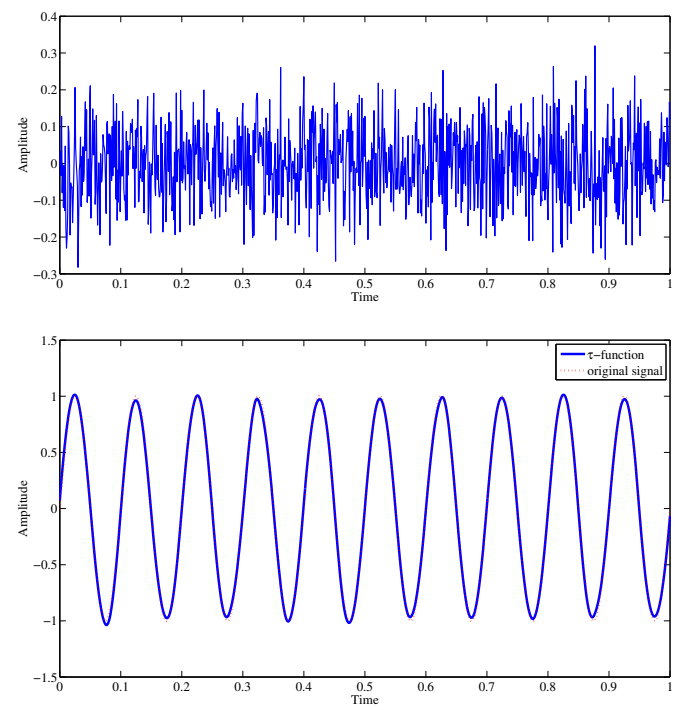

Fig. 2. Using $\tau$-based decomposition to de-noise signal of Fig. 1. Upper figure shows the noise contained in one $\tau$-function. Lower figure shows $\tau$ function containing the low frequency sinusoid compared with the original signal.

\section{DE-NOISING AND DE-TRENDING}

In this section we will describe the application of $\tau$-based decomposition to de-noising and de-trending of synthetic and real-world signals. Figure 1 represents a $10 \mathrm{~Hz}$ sinusoidal signal mixed with additive white gaussian noise having a signal-to-noise ratio (SNR) of $20 \mathrm{~dB}$. Such a signal is quite realistic, for example in the context of electrical noise interference corrupting voltage signals [5]. Elaborate mechanisms for de-noising such a signal based on EMD have been proposed in literature [5]. Using $\tau$-based decomposition, appropriate selection of the value of $\tau$ will isolate the noise in the signal undecomposed in one $\tau$-function, and the low frequency sinusoid will be decomposed into another $\tau$-function. This is shown in Fig. 2, which depicts the noise and the low-frequency sinusoid separated as $\tau$-functions. The lower part of Fig. 2 compares the sinusoid separated from the noise with the original sinusoid. It can be observed that the recovered signal is very close to the original signal. Similarly, Fig. 3 shows the same low-frequency sinusoid recovered as a $\tau$ function when the SNR is decreased to $10 \mathrm{~dB}$ and $5 \mathrm{~dB}$. Even with a low SNR of $5 \mathrm{~dB}$, the de-noised sinusoid is similar to the original $10 \mathrm{~Hz}$ sinusoid, as can be see in the lower part of Fig. 3. As an objective measure of similarity between the de-noised and the original signals, we calculated the values of the correlation coefficient using the windowed RMS values of the de-noised and original signals. The values of the correlation coefficient obtained are $0.996,0.887$ and 0.739 for the $20 \mathrm{~dB}, 10 \mathrm{~dB}$ and $5 \mathrm{~dB}$ cases, respectively.

\section{A. Mode-mixing}

Mode-mixing is a known issue with EMD, and occurs due to intermittency in signals. As described in Section I, intermittency refers to the short-time appearance of particular frequency components in the signal, taking the form of, for example, oscillatory transients. Such a signal is shown in Fig. 4, which is a unit amplitude $10 \mathrm{~Hz}$ sinusoid with low amplitude high frequency $50 \mathrm{~Hz}$ and $100 \mathrm{~Hz}$ sinusoidal components occurring intermittently. Using EMD on this and similar signals leads to mode-mixing, which refers to the situation where an IMF has components of different frequencies due to the fact that 

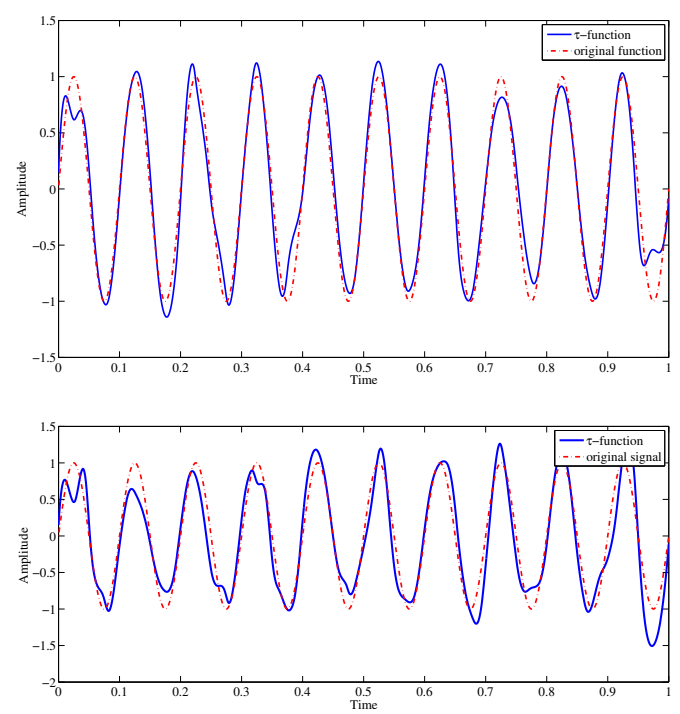

Fig. 3. Upper figure shows the $\tau$-function containing the low-frequency sinusoid recovered when the $\mathrm{SNR}=10 \mathrm{~dB}$. Lower figure shows $\tau$-function containing the low frequency recovered when the $\mathrm{SNR}=5 \mathrm{~dB}$. Both figures compare the de-noised sinusoid with the original $10 \mathrm{~Hz}$ sinusoid.

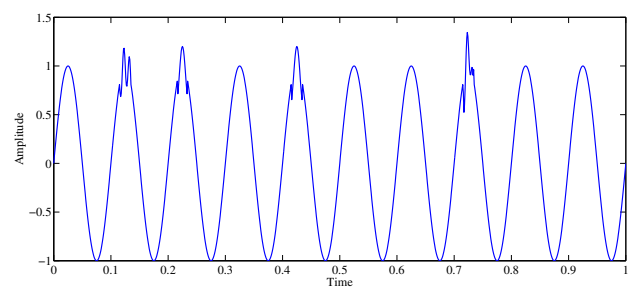

Fig. 4. Low frequency $(10 \mathrm{~Hz})$ signal with intermittently occuring high frequency components

a particular frequency component tracked by an IMF jumps as the intermittent component begins or ends. Elaborate solutions to modemixing have been proposed, such as the use of a masking signal [4]. Also, EEMD, by nature of its algorithm, is also positioned as a solution to mode-mixing, however according to our tests EEMD does not resolve the issue of mode-mixing.

For the signal of Fig. 4, where the intermittency can represent oscillatory transients occurring in many real-world phenomena, an appropriate value of $\tau$ separates the low-frequency sinusoid as a $\tau$-function, and allows the intermittently occurring high-frequency components to pass un-decomposed into another $\tau$-function. This result is shown in Fig. 5. As can be seen from the upper part of Fig. 5, the low-frequency sinusoid extracted as a $\tau$-function is very similar to the original unit amplitude $10 \mathrm{~Hz}$ sinusoid. However, in this case, a value of $\tau$ much higher than that suggested by Eq. 2 has to be used. This behaviour for signals such as that shown in Fig. 4, in which low amplitude high frequency components occur intermittently, can be explained by the nature of the EEMD algorithm when applied to such signals. In the EEMD algorithm, noise is added to the signal to be decomposed. However, for large values of $\tau$, where only few peaks are selected over the whole length of the signal, the envelope formed will introduce spurious low frequency noise in the $\tau$-functions, which will not cancel out, even for low amplitude $\sigma$ of the noise added and a large number $N$ of iterations. This does not happen for smaller values of $\tau$, hence much cleaner $\tau$-functions result.
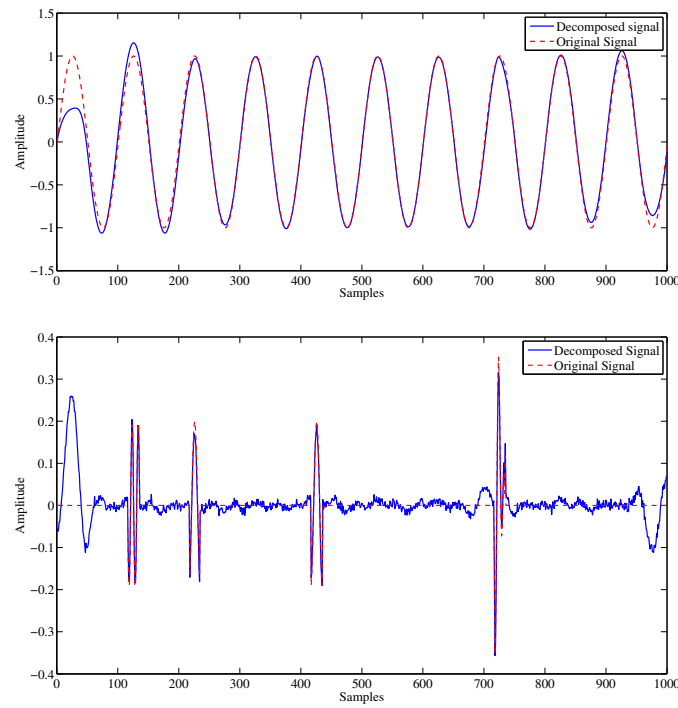

Fig. 5. Decomposing the low freq. signal with intermittently occurring high freq. components of Fig. 4. Upper figure shows $\tau$-function containing low frequency signal, lower figure shows the unwanted high-frequency components isolated in a $\tau$-function. Both figures compare the $\tau$-functions with the original signals as well.

\section{B. Real-world signals}

We have applied $\tau$-based decomposition to real-world signals as well to test the efficacy of the technique. In this context, $\tau$-based decomposition has been used to de-trend EEG signals. Fig. 6 shows the application of $\tau$-based decomposition to EEG signals in order to separate the low-frequency trend from the EEG signals from different channels. In such signals, the interference and the signal of interest are mixed in a non-linear and non-stationary way. The EEG signals are 2500 samples long (10 seconds duration), and have been sampled at 250 samples per second. Fig. 6 (upper half) shows the result of de-trending the EEG signals from four channels. For this, a value of $\tau=12.5$, corresponding to $F=20 \mathrm{~Hz}$ is used. Fig. 6 (lower half) shows the marginal spectrum for the same four channels. The marginal spectrum clearly shows the strength of the method in the low frequency range (below $20 \mathrm{~Hz}$ ), where overlapping of both spectra is common. The $\tau$-based decomposition method filtered out only the interference leaving low frequency activity undisturbed, in contrast to traditional bandpass filtering methods, where frequency content in this range would be cut off. However, this has been achieved with minimal complexity of the method, compared to other approaches for a similar purpose [6].

Automated pathological speech detection using analysis of the speech signals is an active area of research [7]. Pre-processing of the speech signals is often used as part of such an analysis. Fig. 7 shows a 0.5 seconds segment of a pathological speech signal. In order to limit the analysis of the speech signal to a spectrum of $2 \mathrm{kHz}$ only, for example to remove unnecessary noise and interference, $\tau$ based decomposition can be applied to the signal to remove frequency components above the threshold of $2 \mathrm{kHz}$ un-decomposed as a $\tau$ function, and to have the components with frequencies within the $2 \mathrm{kHz}$ range in another $\tau$-function. The speech signal of Fig. 7 is sampled at 25000 samples per second, and a value of $\tau=12.5$ corresponding to $2 \mathrm{kHz}$ is needed for the purpose, according to 


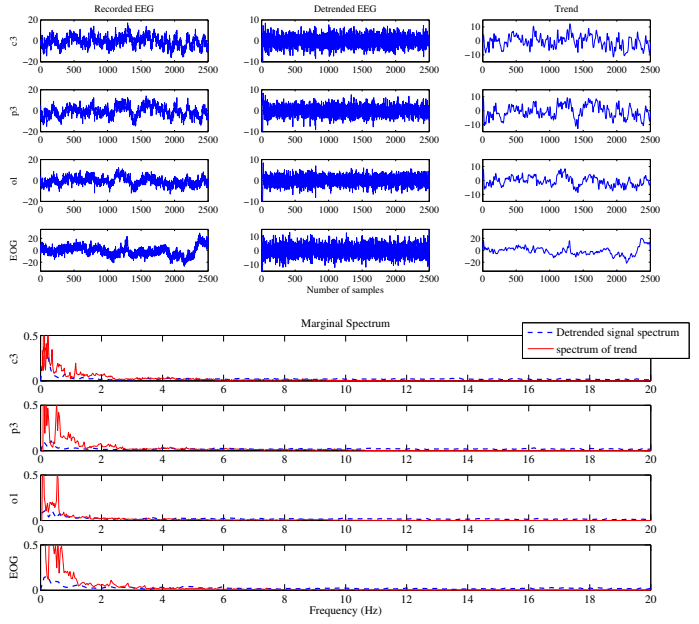

Fig. 6. Using $\tau$-based decomposition to de-trend EEG signals. Upper figure shows detrended EEG signals from four selected channels. Lower figure shows the marginal spectrum; the separation of the spectra is clearly visible.

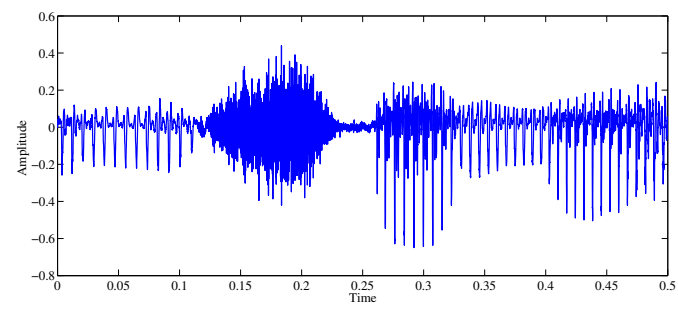

Fig. 7. A 0.5 seconds segment of a pathological speech signal.

the relation $\frac{F_{s}}{\tau}$ presented in Sec. III. The $\tau$-function containing the frequency components above the threshold is shown in the upper part of Fig. 8. The lower part of the same figure contains the denoised signal, which can be used for further analysis. The marginal spectrum of the two $\tau$-functions shown in Fig. 9 clearly shows the separation of the spectra. A closer look at the marginal spectrum of the de-noised signal in Fig. 9 will reveal that there is some spectrum, though negligible, beyond $2 \mathrm{kHz}$. This is in accordance with Eqs. 2 and 3, which means that to completely remove the spectrum beyond the threshold, a value of $\tau$ in accordance with Eq. 2 will have to be used. The same holds true for the marginal spectrum of the EEG signals depicted in Fig. 6.

\section{CONCLUSION}

In this paper we described the $\tau$-based decomposition method which uses a peak selection strategy to decompose a signal into different $\tau$-functions. We also presented the empirically obtained limit relating the value of $\tau$ and the frequency of the components to be decomposed into $\tau$-functions, as shown in Eq. 2. We showed, by using synthetic and real-world signals, how $\tau$-based decomposition can be used to remove noise from signals in a computationally simple way. We also showed that $\tau$-based decomposition can be used to de-noise signals for which application of EMD will result in modemixing. Using EEG and speech signals, it was demonstrated how, using an appropriate value of $\tau$, the signals may be de-trended and de-noised. This demonstrated that the $\tau$-based decomposition method can be applied to cases where interference and signals of interest have significantly different bandwidths, but overlapping spectra, and
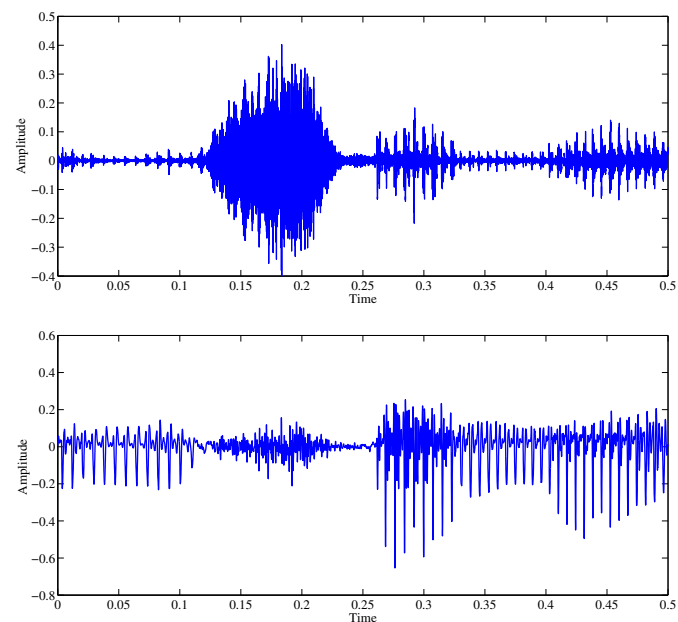

Fig. 8. Using $\tau$-based decomposition to remove noise and interference above a threshold of $2 \mathrm{kHz}$ from speech signal of Fig. 7. Upper figure shows $\tau$ function with components above the threshold, and the lower figure represents the $\tau$-function containing the de-noised signal.

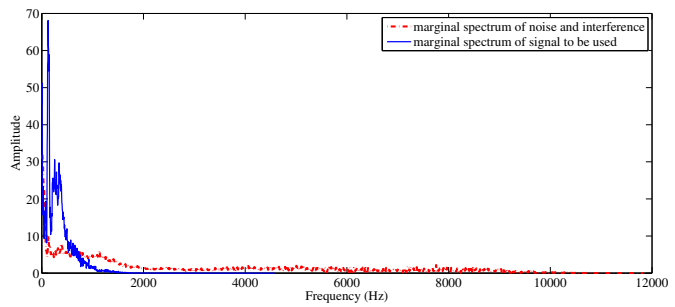

Fig. 9. Marginal spectrum showing the separation of the spectra of the noise and the de-noised signal.

that the $\tau$-based decomposition method represents an effective, but conceptually and computationally simple technique compared to the elaborate approaches mentioned in literature.

\section{REFERENCES}

[1] G. Rilling, P. Flandrin and P. Goncalves, On Empirical Mode Decomposition and its Algorithms, Proceedings of the 6th IEEE/EURASIP Workshop on Nonlinear Signal and Image Processing (NSIP '03), 2003.

[2] Patrick Flandrin, Paulo Goncalves and Gabriel Rilling, "Detrending and denoising with empirical mode decompositions", 12th European Signal Processing Conference, Vienna, Austria, September 6-11, 2004, pp. 15811584.

[3] Zhaohua Wu and Norden E. Huang, "Ensemble Empirical Mode Decomposition: A Noise Assisted Data Analysis Method", Advances in Adaptive Data Analysis, Vol 1, pp. 1-41, 2009.

[4] J.F. Kaiser and R. Deering, "The use of a masking signal to improve empirical mode decomposition", Proceedings of IEEE International Conference on Acoustics, Speech, and Signal Processing (ICASSP '05) , 2005, pp. 485-488.

[5] Vivek Agarwal and Lefteri H. Tsoukalas, "Denoising Electrical Signal via Empirical Mode Decomposition", iREP Symposium- Bulk Power System Dynamics and Control - VII, Revitalizing Operational Reliability, August 19-24, 2007, Charleston, SC, USA.

[6] Md. K. I. Molla and T. Tanaka and T. M. Rutkowski and A. Cichocki, "Separation of EOG Artifacts from EEG Signals using Bivariate EMD", Proceedings of IEEE International Conference on Acoustics, Speech, and Signal Processing (ICASSP '10) , 2010, pp. 562-565.

[7] Behnaz Ghoraani and Sridhar Krishnan, "A Joint Time-Frequency and Matrix Decomposition Feature Extraction Methodology for Pathological Voice Classification", EURASIP Journal on Advances in Signal Processing , 2009. pp. 11 pages. 